Pteridines

Vol. 22, 2011, pp. $13-17$

\title{
Evaluation of Changes in Immune System of Operating Room Personnel by Measurement of Urinary Neopterin Concentrations
}

\author{
Mustafa Baydar', Zeynep Capan², Gozde Girgin ${ }^{3}$, Terken Baydar ${ }^{3}$, Gonul Sahin ${ }^{3}$ \\ ${ }^{1}$ Anesthesiology and Reanimation Clinic, Numune Hospital; ${ }^{2}$ Anesthesiology and Reanimation Clinic, Ankara \\ Hospital; ${ }^{3}$ Department of Toxicology, Faculty of Pharmacy, Hacettepe University; Ankara, Turkey
}

Accepted: 2011/04/08

\begin{abstract}
Today it is known that neopterin is mainly produced by activated macrophages and a marker of immune activation and macrophage activity. Increased neopterin concentrations are observed in diseases related to cellular immunity including occupational pathologies.

The major goal of the present study was to evaluate the possible alteration of neopterin levels in operating room personnel, and also to show whether screening of neopterin may be useful to monitor the effect of occupational anesthetic exposure on the cellular immune system.

Therefore, urinary neopterin to creatinine levels in both, exposed workers $(n=40)$ and healthy volunteers $(\mathrm{n}=30)$, were measured by using high-performance liquid chromatography. At the same time, the correlation among urinary neopterin levels and working years, age, and smoking status were evaluated.

Compared to controls, urinary neopterin levels in the exposed group were increased (controls: $85 \pm 16 \mu \mathrm{mol} / \mathrm{mol}$ creatinine, workers: $151 \pm 39 \mu \mathrm{mol} / \mathrm{mol}$ creatinine; $\mathrm{p}<0.05)$.

The findings suggest that the follow up of neopterin levels may have diagnostic value in possible occupational exposure-related immune system disorders. Moreover, its biological monitoring should be performed in workplaces for clinical diagnosis and prognosis.
\end{abstract}

Key words: $\quad$ operating room personnel, neopterin

\section{Introduction}

Occupational health and safety is a field that involves many disciplines which are dealing with workplace problems. Chemicals are found in many if not all workplaces and require careful attention to avoid overt acute poisoning as well as in the longer term, more insidious adverse health effects that may occur $(1,2)$. Among the workplaces, operating theatres have a huge importance, since they are inevitably very common everywhere. Operating room personnel such as surgeons, anesthesiologists, technicians and nurses are exposed to a variety of noxious agents in their workplace. Of particular importance are various infectious agents, trace amounts of anesthetic gases and cleaning substances and solvents $(2,3)$. In spite of the attempt to reduce the risk by modern air-conditioning and -scavenging systems, the levels of inhalational anesthetics in air of operating rooms may often exceed those limits stated in national guidelines and may cause chronic exposure in workers (4). As a result of this, many untoward effects such as spontaneous abortion, mood disorder, lethargy, depression, anemia, hepatitis, reduction in protective systems of the organism, or depletion of neuro-respiratory activity, etc. may occur $(4,5)$. The possible contaminants which may be present in air of the operation rooms may also produce oxidative stress, sensitization, inflammation, and modulation of the immune system $(2,3)$. Therefore, routine monitoring of indoor air quality for workplaces and evaluation of health status of workers are useful and important to prevent hazards and reduce risks. Biomarkers of exposure and effect are becoming of increased interest for this purpose $(1,2)$. Nowadays, particular attention is paid to noninvasive early markers of damage such as neopterin which is a major unconjugated pteridine. The pro-inflammatory mediator neopterin is regarded as a early biochemical marker 
of cell-mediated immunity. It is primarily produced by activated monocytes/macrophages from guanosine triphosphate (GTP) via GTP-cyclohydrolase I $(6,7)$. Interferon- $\gamma($ IFN- $\gamma$ ) has been observed to up-regulate the synthesis and release of neopterin (8), while tumor necrosis factor- $\alpha$ (TNF- $\alpha$ ) and endotoxins can costimulate neopterin production. Neopterin seems useful as a parameter of activation of the cell-mediated immune system $(9,10)$.

Therefore, the present study was undertaken in order to evaluate the immune status in operating room workers by measuring urinary neopterin levels. In addition to this, the neopterin results of urine samples obtained from two different hospitals were compared.

\section{Materials and Methods}

\section{Subjects}

The operation room personnel group consisted of forty people (mean age, $34 \pm 8$ years; 19 female and 21 male) including surgeons, anesthesiologists, and anesthesia technicians who work in surgery and anesthesiology clinics of Numune Hospital $(n=20)$ and Ankara Hospital $(\mathrm{n}=20)$ in 2008-2009. A questionnaire was designed for each subject to yield information on sex, age, occupational history and general health-status. The unexposed group as a control group was 30 healthy subjects (mean age $29 \pm 6$ years; 21 female and 9 male). The controls did not work in the operating rooms, did not have any systemic diseases and were not receiving any medication during sample collection period.

The operation room personnel was divided into two subgroups according to working duration: group 1: from three months to 5 years ( $\leq 5$ years), and group 2: 10 years and older ( $\geq 10$ years). The mean ages of the groups 1 and 2 were $29 \pm 3$ and $42 \pm 6$ years, respectively.

The principles of the Ethical Committees of Numune and Ankara Hospitals according to the Helsinki Declaration were followed during the whole study.

\section{Sampling}

Urine samples were collected in the early morning and kept from direct light, and stored at $-20^{\circ} \mathrm{C}$ until analysis.

\section{Measurements}

Urinary neopterin levels were determined by highperformance liquid chromatography (HPLC) using a fluorescence detector (HP, Agilent1100) at a wavelength of $353 \mathrm{~nm}$ for excitation and $438 \mathrm{~nm}$ for emission, as described before $(11,12)$. Creatinine levels were detected simultaneously by HPLC using an ultraviolet detector (HP, Agilent1100) at the wavelength of $235 \mathrm{~nm}$. The neopterin levels were given as micromoles of neopterin per mole of creatinine $(\mu \mathrm{mol}$ neopterin/mol creatinine).

\section{Statistics}

The results were expressed as mean values with standard deviation (mean $\pm \mathrm{SD}$ ). Because there was no normal distribution in all the data sets analyzed, nonparametric statistics were applied. Differences among groups were evaluated with Kruskal-Wallis analysis of variance while Mann-Whitney U test was used to compare the differences between two independent groups. Spearman correlation test was used to investigate the correlations of the parameters. $\mathrm{p}<0.05$ was considered to indicate statistical significance.

\section{Results}

The urinary neopterin levels were $84.8 \pm 15.8$ and $151.3 \pm 39.0 \mu \mathrm{mol} / \mathrm{mol}$ creatinine in the 30 controls and the 40 workers (Figure 1$)(p=0.001)$. Thus, the mean value of urinary neopterin levels in workers was $78 \%$ higher than those of controls.

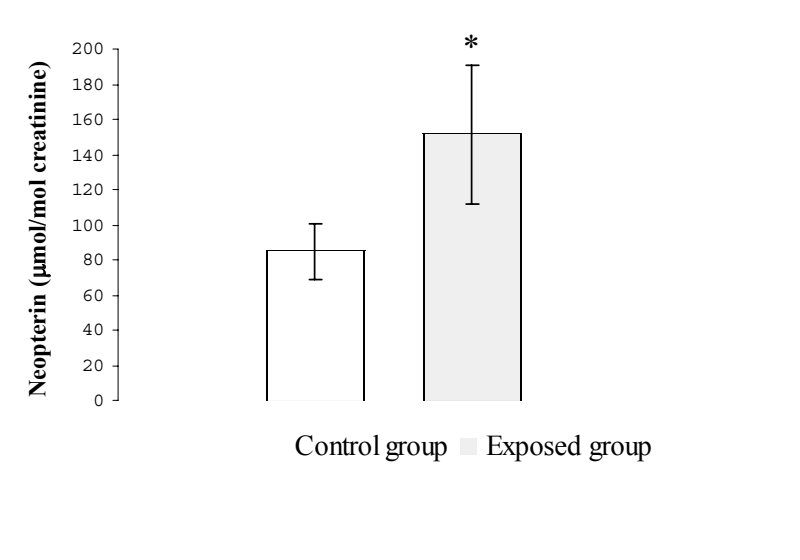

Figure 1. Urinary neopterin concentrations in controls $(n=30)$ and workers exposed to anesthetics $(n=40)$. $* \mathrm{p}<0.05$; compared to the control group.

Because of the collection of the samples from two different hospitals (Numune and Ankara), the results of neopterin concentrations were tested if there were any differences between the two hospitals but this was not the case $(p>0.05)$. On the other hand, when the results of each hospital were compared separately with the corresponding control group the differences were statistically different (both $\mathrm{p}<0.05$ ), see Table 1 .

When the working duration period was considered, the results in both of the subgroups were significantly 
Table 1. Urinary neopterin concentrations in controls and workers in the two hospitals $* \mathrm{p}<0.05$; versus controls.

$\begin{array}{lccc}\text { Groups (n) } & \text { Mean } \pm \text { SD } & \text { Minimum } & \text { Maximum } \\ \text { Hospital 1 (20) } & 151.6 \pm 42.7^{*} & 101.6 & 268.9 \\ \text { Hospital 2 (20) } & 151.0 \pm 36.5^{*} & 100.3 & 237.7 \\ \text { Controls (30) } & 84.8 \pm 15.8 & 60.4 & 119.0\end{array}$

Table 2. Effect of duration period on urinary neopterin concentrations, workers grouped into those with $\leq 5$ and $\geq 10$ years of working periods $* \mathrm{p}<0.05$; versus controls.

\begin{tabular}{lccc}
\hline \multirow{2}{*}{ Groups } & \multicolumn{3}{c}{ Neopterin $(\mu \mathrm{mol} / \mathbf{m o l}$ creatinine) } \\
\cline { 2 - 4 } & Mean $\mathbf{\text { SD }}$ & Minimum & Maximum \\
\hline$\leq \mathbf{5}$ years (22) & $158.2 \pm 47.6^{*}$ & 101.6 & 268.9 \\
\hline$\geq \mathbf{1 0}$ years (18) & $143.0 \pm 24.3^{*}$ & 100.3 & 182.8 \\
\hline Controls (30) & $84.8 \pm 15.8$ & 60.4 & 119.0 \\
\hline
\end{tabular}

higher than those of the controls as shown in Table 2 (both $\mathrm{p}<0.05$ ). However, there was no difference between the two subgroups in urinary neopterin levels ( $p>0.05$ ). When comparing the urinary neopterin levels with the working-time it was observed that a longer working period was weakly associated with lower neopterin excretion, but the correlation was not statistically significant $\left(r_{\mathrm{s}}=-0.192 ; \mathrm{p}=0.286\right)$.

It was found that there was no effect of gender on urinary neopterin levels $(p>0.05)$ and there was not any correlation between age and neopterin concentration $\left(r_{s}=-0.102 ; p=0.573\right)$. However, when considering minimum and maximum ages in both of the control and exposed groups, the urinary neopterin level in the youngest worker ( 22 years-old) was $53 \%$ higher than its control aged 22, while the percentage of neopterin elevation in the oldest worker (both, 42 years) was $27 \%$.

\section{Discussion}

In parallel to modulation of cellular immune system, the alterations in neopterin levels are well-documented in many diseases (11-17). Neopterin concentrations in body-fluids increase in viral, bacterial, and parasitic infections. Furthermore, states of the diseases, elective surgery, and severe trauma also cause an increase in levels of biomarker neopterin, which is biologically stable and can be easily quantified in noninvasive biological samples (13-16). Stressors such as some environmental and occupational xenobiotics can also cause changes in neopterin status $(18,19)$. Operating theatre staff can be occupationally exposed to a variety of stress factors including biological agents and waste and residue of chemicals. Long term occupational exposure to low levels of inhalational anesthetics is thought to have adverse effects on the health of exposed personnel (5). The possible noxious agents in anesthetic work place may also affect many organs including immune system in operating room workers $(3,5)$. The studies about the possible alteration of immune system by measuring neopterin levels are limited $(3,20)$. One of the few studies was performed by Gruber et al. in order to examine urinary neopterin levels in anesthetic work place workers, and they have not found any elevation urinary neopterin levels when compared to the normal ranges established previously in healthy controls (3). In the present study, we have found that there was a statistically significant difference between the mean neopterin concentrations in controls and the exposed group $(\mathrm{p}<0.05)$. The inconsistency between the results of the two studies might have resulted from the conditions of the operating rooms, types of anesthetic techniques or used anesthetics, and etc. Despite of opposites on effect of smoking in neopterin levels were reported $(3,21,22)$, in our 
previous (18) and the present studies we have not observed any effect of smoking in neopterin excretion.

As observed before $(11,12)$, age and gender did not cause any alteration in urinary neopterin concentration in the present study. We also found that working duration has slightly led to reduction of urinary neopterin levels, but the difference was not statistically significant. This lower neopterin with longer working duration would agree rather well with the result of the Gruber et al. study where older anesthesiologists presented with lower neopterin levels (3). It can be expected that the duration of exposure to air of anesthetic work place may play a role in immune system stimulation of personnel as well as substances in the operating theatre. However, the influence was not significant in the individuals in our study but the slightly diminished neopterin levels in the group exposed to air of operation work place for a long time (over 10 years) might be explained as development of adaptation to chemical stressors. In other words, immune system has adapted to continuous occupational exposures with increasing working duration.

In conclusion, our results strongly suggested that the workers in operating rooms should be routinely monitored with early biomarkers such as neopterin levels. More detailed researches in this field are needed.

\section{Declaration of Conflicting Interests}

The authors report no conflicts of interest. The authors alone are responsible for the content and writing of the paper.

\section{References}

1 Occupational medicine-Interface with toxicology, Chapter 16, in Occupational Toxicology, W.O. Phoon, Neil H. Stacey (eds). Taylor and Francis, London, 1995, 295-304.

2 Thorne Ps, Occupational Toxicology, Chapter 33, in Casarett and Doull's Toxicology: The Basic Science of Poisons, CD Klaassen (ed). 6th edition, McGraw-Hill Companies, Inc., 2001, 1123-1140.

3 Gruber G, Lirk P, Amann A, Keller C, Schobersberger W, Hoffmann G, Fuchs D, Rieder J. Neopterin as a marker of immunostimulation: an investigation in anaesthetic workplaces. Anaesthesia 2002; 57: 747-750.

4 Irwin MG, Trinh T, Yao CL. Occupational exposure to anaesthetic gases: a role for TIVA. Expert Opin Drug Saf 2009; 8 (4): 473-483.
5 Byhahn C, Wilke HJ, Westphal K. Occupational exposure to volatile anaesthetics: epidemiology and approaches to reducing the problem. CNS Drugs 2001; 15 (3): 197-215.

6 Fuchs D, Weiss G, Reibnegger G, Wachter H. The role of neopterin as a monitor of cellular immune activation in transplantation, inflammatory, infectious, and malignant diseases. Crit Rev Clin Lab Sci 1992; 29: 307-341.

7 Wirleitner B, Reider D, Ebner S, Guenther B, Widner B, Jaeger M, Schennach H, Romani N, Fuchs D. Monocyte-derived dendritic cells release neopterin. J Leukocyte Biol 2002; 72: 1148-1153.

8 Huber C, Batchelor JR, Fuchs D, Hausen A, Lang A, Niederwieser D, Reibnegger G, Swetly P, Troppmair J, Wachter H. Immune response-associated production of neopterin. Release from macrophages primarily under control of interferongamma. J Exp Med 1984; 160: 310-316.

9 Werner-Felmayer G, Werner ER, Fuchs D, Hausen A, Reibnegger G, Wachter $H$. Tumour necrosis factor-alpha and lipopolysaccharide enhance interferon-induced tryptophan degradation and pteridine synthesis in human cells. Biol Chem Hoppe Seyler 1989; 370: 1063-1069.

10 Widner B, Wirleitner B, Baier-Bitterlich G, Weiss G, Fuchs D. Cellular immune activation, neopterin production, tryptophan degradation and the development of immunodeficiency. Arch Immunol Ther Exp (Warsz) 2000; 48: 251-258.

11 Baydar T, Yuksel O, Sahin TT, Dikmen K, Girgin G, Sipahi H, Kurukahvecioglu O, Bostanci H, Sare M. Neopterin as a prognostic biomarker in intensive care unit patients. J Crit Care 2009; 24: 318321.

12 Sahin TT, Yuksel O, Sipahi H, Girgin G, Dikmen K, Azili C, Taneri F, Baydar T. Urinary neopterin in patients with thyroid disorders. J Endocrinol Invest 2009; 32 (2): 147-149.

13 Berdowska A, Zwirska-Korczala K. Neopterin measurement in clinical diagnosis. J Clin Pharm Ther 2001; 26: 319-329.

14 Murr C, Widner B, Wirleitner B, Fuchs D. Neopterin as a marker for immune system activation. Curr Drug Metab 2002; 3: 175-187.

15 Ruokonen E, Ilkka L, Niskanen M, Takala J. Procalcitonin and neopterin as indicators of infection in critically ill patients. Acta Anaesthesiol Scand 2002; 46: 398-404.

16 Ip M, Rainer TH, Lee N, Chan C, Chau SSL, Leung W, Leung MF, Tam TK, Antonio GE, Lui G, Lau TK, Hui DSC, Fuchs D, Renneberg R, Chan PKS. Value of serum procalcitonin, neopterin, and C-reactive protein in differentiating bacterial from viral etiologies in patients presenting with lower 
respiratory tract infections. Diagn Microbiol Infect Dis 2007; 59: 131-136.

17 Murr C, Fuith LC, Widner B, Wirleitner B, BaierBitterlich G, Fuchs D. Increased neopterin concentrations in patients with cancer: indicator of oxidative stress? Anticancer Res 1999; 19 (3A): 17211728.

18 Altindag ZZ, Baydar T, Isimer A, Sahin G. Neopterin as a new biomarker for the evaluation of occupational exposure to silica. Int Arch Occup Environ Health 2003; 76: 318-322.

19 Ulker OC, Yucesoy B, Durucu M, Karakaya A. Neopterin as a marker for immune system activation in coal workers' pneumoconiosis. Toxicol Indust Health 2007; 23 (3): 155-160.
20 Leichtfried V, Putzer G, Perkofer D, Schobersberger W, Benzer A. Circadian neopterin profiles during a single 24-hour shift in anaesthetists. Pteridines 2010; 21 (2): 48-49.

21 Diamondstone LS, Tollerud DJ, Fuchs D, Wachter H, Brown LM, Maloney E, Kurman CC, Nelson DL, Blattner WA. Factors influencing serum neopterin and beta 2-microglobulin levels in a healthy diverse population. J Clin Immunol 1994; 14: 368-374.

22 Schennach H, Murr C, Gachter E, Mayerbach P, Schönitzer D, Fuchs D. Factors influencing serum neopterin levels in a population of blood donors. Clin Chem 2002; 48 (4): 643-645. 